\title{
MÉXICO Y LA COOPERACIÓN INTERNACIONAL PARA EL DESARROLLO, 2012-2018
}

\author{
MEXICO AND INTERNATIONAL COOPERATION \\ FOR DEVELOPMENT, 2012-2018
}

\section{LE MEXIQUE ET LA COOPÉRATION INTERNATIONALE POUR LE DÉVELOPPEMENT, 2012-2018}

\author{
Juan Pablo Prado Lallande \\ Benemérita Universidad Autónoma de Puebla \\ juanp.prado@correo.buap.mx
}

Resumen: Al inicio de su mandato, el presidente Enrique Peña Nieto estableció que la política exterior se basaría en la cooperación internacional para el desarrollo (CID). Ante ello, el objetivo de este artículo es analizar las características de orden institucional y fácticas más definitorias de la CID ejercida durante esa administración, poniendo énfasis en el papel de la Amexcid como la instancia responsable de coordinar esta actividad. El artículo concluye que, en los respectivos contextos nacional e internacional -entendidos como factores estructurales de la CID- que oscilaron de lo favorable a lo convulso, la CID del sexenio 2012-2018 se caracterizó por alentadores avances, aunque también por inconsistencias que dificultaron que se constituyera en un activo de la política exterior, conforme a las expectativas despertadas.

Palabras clave: cooperación internacional para el desarrollo; cooperación Sur-Sur; política exterior; Amexcid; Enrique Peña Nieto.

Abstract: At the beginning of his term, President Enrique Peña Nieto declared that foreign policy would be based on a principle of international cooperation for development (ICD). The objective of this article is to analyze the most salient institutional and factual characteristics of ICD as exercised during this administration, emphasizing the role of Amexcid as the entity responsible for coordinating this activity. The article concludes that, in the respective national and international contexts -understood as structural factors of the ICD- which ranged from favorable to turbulent, the ICD of the 2012-2018 administration was characterized by encouraging progress, but also by a lack of robustness 
which made it difficult for it to become a foreign policy asset, in line with the expectations aroused.

Keywords: international cooperation for development; South-South cooperation; foreign policy; Mexico; Amexcid; Enrique Peña Nieto.

\section{Traducción de Gonzalo Celorio Morayta}

RÉsumÉ: Au début de son mandat, le président Enrique Peña Nieto a établi que sa politique étrangère serait fondée sur la coopération internationale pour le développement. L'article examine les facteurs institutionnels et les circonstances qui ont le plus influencé cette coopération le long de la période analysée; on insiste sur le rôle de l'Agence mexicaine de coopération internationale pour le développement (Amexcid), en tant que responsable de la coordination dans ce domaine. La conclusion est que, sous les effets structurels d'une atmosphère nationale et internationale qui passait du calme à l'agitation, la coopération pour le développement a fait des progrès encourageants, même si un certain manque de solidité l'a empêché de devenir un élément de la politique étrangère à la hauteur de l'espoir éveillé.

Mots clefs: coopération internationale pour le développement; coopération Sud-Sud; politique étrangère; Mexique; Amexcid; Enrique Peña Nieto.

Traducción de Bernardo MABIRE

Fecha de recepción: diciembre de 2018

Fecha de aceptación: febrero de 2019 
Cooperamos porque hemos querido, pero nuestra cooperación voluntaria nos crea obligaciones que no habíamos deseado.

EMile Durkheim

\section{$\mathrm{E}$}

$\mathrm{N}$ un mundo cada vez más competitivo, aquellos países que pretenden instrumentar una política exterior activa recurren a la cooperación internacional para el desarrollo (CID), a efecto de ampliar su presencia en Estados y regiones de su predilección. En este tenor, desde hace al menos siete décadas, sea en su dimensión oferente, receptora o dual, México constituye un interesante referente latinoamericano.

De forma coincidente con lo anterior, el gobierno del presidente Enrique Peña Nieto (2012-2018), mediante su Plan Nacional de Desarrollo (PND) propuso hacer del país "un actor con responsabilidad global", 1 para lo cual se estableció que "la política exterior se basará en la CID...".2 Ello, entre otras circunstancias, generó expectativas de reforzar la CID mexicana en sus aristas política, institucional y financiera, a efecto de que su ejercicio constituyera un mecanismo más vigoroso en esa administración.

Fue así como en dicho sexenio, la Secretaría de Relaciones Exteriores (SRE), a través de su Agencia Mexicana de Cooperación Internacional para el Desarrollo (Amexcid), llevó a cabo distintos esfuerzos tendientes a alinear de mejor manera la cooperación mexicana con los objetivos de su política exterior, en concordancia con la Ley de Cooperación Internacional para el Desarrollo (LCID), promulgada en 2011.

De cara a este panorama, el objetivo de este artículo es analizar las características de orden institucional y fácticas más definitorias de la cID ejercida durante el gobierno de Peña Nieto, poniendo énfasis en el papel de la Amexcid como

${ }^{1}$ Gobierno de la República, Plan Nacional de Desarrollo 2013-2018, México, Gobierno de la República, 2013, p. 91.

${ }^{2}$ Ibid., p. 991. 
la instancia que, conforme a la citada ley, es la responsable de coordinar esta actividad en la esfera federal.

Para satisfacer tal propósito, el texto se divide en cuatro apartados además de esta introducción. El primero examina la CID desde sus distintas variables, enfatizando la herencia que el gobierno de Peña Nieto recibió en este rubro de su antecesor. El segundo apartado revisa los objetivos, los ejes programáticos y los planteamientos políticos con que se pretendió moldear la CID de 2013 a 2018. El tercero estudia las acciones de mayor significación en este ámbito, tomando como referente las particularidades de la Amexcid y los recursos que se le confirieron. Con base en ello, el cuarto apartado analiza los resultados más sobresalientes en la materia durante el sexenio estudiado.

Las consideraciones finales avalan la hipótesis de esta investigación, la cual sostiene que la política exterior que instrumentó Peña Nieto dependía de factores internos y externos, que a partir de 2017 no fueron propicios y afectaron los factores estructurales que moldean el ejercicio de la CID, lo que explica el debilitamiento de la capacidad gubernamental para instrumentarla a la altura de las aspiraciones planteadas. Tal situación, sin dejar de valorar los logros alcanzados, restringió el potencial político y la capacidad institucional y financiera de la CID mexicana como un instrumento clave de la política exterior, la cual en este sexenio registró avances, aunque también inconsistencias.

\section{LA HERENCIA}

La CID complementa capacidades nacionales a efecto de impactar de manera sostenible en los factores de los cuales depende el nivel de vida de los habitantes del país socio. Si bien esto es cierto, en realidad sus propósitos van más allá de este referente ético, en especial, cuando se utiliza como herramienta de política exterior. Esto lleva a que, desde la perspectiva político-diplomática, la instrumentación de ac- 
tos cooperativos no sea un fin en sí mismo, sino más bien un medio para atender de forma armónica distintos propósitos gubernamentales de mayor calado.

De ahí que los objetivos de la CID, en cada caso, pretenden satisfacer el interés nacional del proveedor, apoyando a determinados aliados en función de criterios geopolíticos (fundamento realista), intentando atender necesidades en países socios con una visión solidaria (perspectiva constructivista) y buscando cumplir propósitos globales mediante normas e instituciones internacionales, como la Agenda 2030 y sus Objetivos de Desarrollo Sostenible (óptica liberal-institucional).

Estos planteamientos permiten aseverar que la CID se alimenta de la conjunción incluyente de varios criterios de asignación, en función de los propósitos en boga, ${ }^{3}$ que pueden sintetizarse en conseguir réditos afines a los preceptos del poder suave. ${ }^{4}$ Empero, si bien puede propiciar óptimos resultados en éstos y otros rubros, practicar esta cooperación no siempre conlleva el logro automático de las metas planteadas, en razón de que su eficacia radica en al menos tres factores estructurales.

El primero se refiere a las particularidades del contexto doméstico e internacional de su ejercicio, de lo cual se desprenden los objetivos, las prioridades temáticas y la jerarquización de los beneficiarios; el segundo, la voluntad política para llevarla a cabo, ya sea con mayor o menor énfasis, y, el tercero, las experiencias previas y la capacidad legal, institucional y financiera del gobierno que la instrumenta.

En este dinámico contexto y sujeto a tales factores estructurales, México, al igual que otros países emergentes, intenta ejercer un rol relevante que le permita posicionarse en esta parcela del acontecer mundial. El activismo mexicano en

${ }^{3}$ Carlos R. S. Milani, Solidariedade e Interesse: motivações e estratégias na cooperação internacional para o desenvolvimento, Curitibba, Brasil, Appris Editora, 2018, p. 198.

${ }^{4}$ Joseph S. Nye Jr., El poder suave. La clave del éxito en la política internacional, México, Universidad Iberoamericana, 2016. 
este rubro de su política exterior no es reciente, sino que se remonta a décadas atrás.

La década de los ochenta, en razón del desafío centroamericano de aquella época, catapultó la imagen de México como proveedor de cooperación Sur-Sur (CSs) en esa región; un desafiante contexto que dinamizó el ejercicio de la CID como componente prioritario -en esos momentos- de su actividad externa. En 1988, cuando se establecieron en el artículo 89 de la Constitución los principios normativos de la política exterior, la cooperación internacional para el desarrollo formó parte de tales referentes.

En materia de institucionalización de la CID en México, tras un cúmulo de experiencias en la SRE y gracias a una iniciativa de ley propuesta por Rosario Green, senadora del Partido Revolucionario Institucional (PRI), el 16 de abril de 2011 se consiguió que entrara en vigor la LCID. Este novedoso lineamiento jurídico incluye una batería de disposiciones destinadas a concederle al gobierno mexicano los instrumentos necesarios para alinear de manera más eficaz la CID con la política exterior y el desarrollo nacional.

Dicho ordenamiento, que constituye el pilar legal, además de la Amexcid y su Consejo Consultivo (su brazo coordinador), dispone la creación del Programa de CID (Procid), que le da sustento programático. Incluye también el Sistema Nacional de Información (InfoAmexcid) y un Registro Nacional (Rencid), como herramientas estadísticas y de rendición de cuentas, así como el Fondo Nacional de CID (Foncid), que proporciona un marco financiero.

Sin embargo, el presidente Felipe Calderón consideró que la ley era una afrenta política proveniente de un partido opositor, en un tema de casi exclusiva potestad del Poder Ejecutivo, como es la política exterior, y, además, en un tópico secundario para su administración, por lo que no la respaldó con suficiente empeño. ${ }^{5}$ Por ello, si bien la Amexcid se

${ }^{5}$ Patricia Espinosa Cantellano, titular de esa cartera, sobre por qué la Amexcid se creó a imagen y semejanza de la Unidad de Relaciones Econó- 
creó el 28 de septiembre de 2011, la Unidad de Relaciones Económicas y Cooperación Internacional (URECI) se configuró a imagen y semejanza de su instancia antecesora. Ante tal situación Rosario Green aseveró, en 2012, que desde su punto de vista la Amexcid distaba "mucho de consolidarse y cumplir con las exigencias de la ley que le dio origen". ${ }^{6}$

Asimismo, otros preceptos de la ley no se atendieron, como fue el caso del Procid, que aunque se elaboró, no entró en vigor. De manera semejante, no se concretaron el Rencid, el InfoAmexcid ni el Foncid. En síntesis, a finales de 2012, Peña Nieto heredó de su antecesor un vasto acervo en la materia acumulado en décadas de trayectoria, junto con una nueva ley y una agencia impulsadas por su propio partido, el PRI, que se hallaban vigentes, aunque con niveles de operación limitados.

\section{LA EXPEGTATIVA}

La llegada del PRI al poder a finales de 2012 generó expectativas de que, tal y como lo hicieran sus legisladores en el Congreso, el nuevo gobierno federal posicionaría la cID como un componente más consolidado de una renovada política

micas y Cooperación Internacional (URECI), comentó: "Lo que pasó, sinceramente, es que no hubo la decisión de asignar recursos para la agencia, para crear una estructura de la agencia, que debería haber sido el caso, la verdad [...] Como gobierno, oponerse a la aprobación de la ley estaba muy difícil, políticamente era muy difícil. Entonces, la manera de destrabar la objeción, de forma pragmática, fue bueno, la echamos a andar con esta parte de la cancillería que ya existe [la URECI]. Ello con el deseo y la esperanza de que en el camino se pueda ir generando la posibilidad de crear las plazas para la agencia, de separar en efecto por ejemplo la parte de relaciones económicas bilaterales en las áreas regionales de la cancillería y dejar realmente la parte de cooperación" (Juan Pablo Prado Lallande, "La cooperación internacional para el desarrollo de México", Revista Mexicana de Ciencias Políticas y Sociales (2014), núm. 222, p. 72).

${ }^{6}$ Rosario Green, "Política exterior de Estado con los tres órdenes de gobierno", El Universal, 1 de agosto de 2012. 
exterior, honrando en consecuencia los preceptos de "su" ley en la materia. Por ende, y como se verá en seguida, el PND 2013-2018 recategorizó la cooperación internacional para el desarrollo como instrumento proactivo de la política exterior mexicana, que propugnaba hacer del país "un actor con responsabilidad global". ${ }^{7}$

Testimonio de ello es que en el PND la CID se definió como uno de los cuatro pilares en los que se basaría la política exterior del sexenio. De hecho, en el primer punto del plan de acción para consolidar el papel de México en el mundo, se aseveró de forma enfática: "la política exterior se basará en la CID". ${ }^{8}$ En sintonía con tal postulado, el 30 de abril de 2014 el gobierno federal hizo historia al publicar el Procid, por vez primera y conforme lo estipula la LCID. A la luz de este trascendente avance, y como un reflejo de la renovada relevancia del binomio que forman la política exterior y la CID, en 2014 el secretario de Relaciones Exteriores expresó que ésta "es un instrumento útil para lograr que México alcance una mayor presencia internacional".9

Un acontecimiento representativo del mayor peso político del tema fue la celebración, en abril de ese año en suelo mexicano, de la Primera Reunión de Alto Nivel de la Alianza Global para la Cooperación Eficaz al Desarrollo (AGCED); ${ }^{10}$

${ }^{7}$ Ibid., p. 91.

${ }^{8}$ Ibid., p. 991.

${ }^{9}$ José Antonio Meade Kuribreña, "México y la cooperación internacional para el desarrollo", Revista Mexicana de Política Exterior, (2014), núm. 102, p. 5.

${ }^{10}$ La AGCED reúne de manera informal a actores estratégicos en este ámbito a escala global. El liderazgo mexicano en ese encuentro no impidió que China e India se ausentaran del encuentro en cuestión, lo cual desdibujó el papel de México como "puente entre el norte y el sur", aunque ciertamente le dio proyección mundial, al ser sede de esta reunión, con rango ministerial. Véase Juan Pablo Prado Lallande, “Mexico's Role in Development Cooperation: Bridging North and South", en Rahul Chandran y Hannah Cooper (eds.), Meeting the Challenges of the 2030 Agenda: Alternative Forms of Development Cooperation and the United Nations, Tokyo, United Nations University, 2015. 
relevante acto que fue inaugurado por el presidente de la República. En esa ocasión, Peña Nieto se refirió explícitamente a la CID al señalar que

México es un país solidario y generoso que ha hecho de la cooperación y colaboración económica, la asistencia humanitaria y el esfuerzo compartido por un mundo mejor, un testimonio claro de su responsabilidad global. Somos un país que ha creado su propia Agencia de CID, convencidos de que podemos coadyuvar a superar la pobreza y reducir las brechas de desigualdad que aún dividen a los habitantes del planeta. $^{11}$

Así las cosas, con éstos y otros planteamientos que configuraron un ambiente favorable para mejorar la trayectoria nacional en la materia, motivó a los altos funcionarios de la agencia a resaltar "la importancia de consolidar la CID como una política de Estado, y la Amexcid como una referencia a nivel regional y global". ${ }^{12}$ En síntesis, en esos momentos, los factores estructurales de la cooperación internacional se mostraban favorables para que el gobierno en turno estuviese en capacidad de impulsar una dinámica política exterior y, así, hacer efectivos la LCID y los preceptos correspondientes contenidos en el PND 2013-2018.

\section{LOS HECHOS}

Los institucionalistas aseveran que la estructura de las agencias de cooperación internacional, organismos multilaterales, etc., determinan la capacidad de estos entes para conseguir sus objetivos y, por ende, satisfacer las expectativas generadas

${ }^{11}$ Enrique Peña Nieto, "Discurso de inauguración", Gobierno de la República - SRE - Amexcid - GPED, 2014, Primera Reunión de la AGCED, México, 2016, p. 16.

12 Daniela Borbolla Compeán, "La construcción institucional de la Amexcid”, Revista Mexicana de Política Exterior, 2014, núm. 102, p. 70. 
en el ámbito de su incumbencia. ${ }^{13}$ De ahí que la revisión del estado de la Amexcid en los últimos seis años facilita la comprensión de las acciones y alcances de la colaboración mexicana en este periodo. Sin embargo, dado que, a fin de cuentas, los modelos institucionales de la CID en México y en otros países dependen en buena medida de decisiones políticas, el análisis a este respecto debe iniciarse por el nombramiento del director ejecutivo de la Amexcid, designado por la presidencia de la República, a propuesta del titular de la SRE.

La persona elegida para ostentar el cargo a partir del $1^{\circ}$ de enero de 2013 y durante casi tres años fue Juan Manuel Valle Pereña, exdirector de la Unidad de Banca, Valores y Ahorro de la Secretaría de Hacienda y Crédito Público (SHCP). Lo anterior no sería pertinente si se omite que la LCID restringe la elección del encargado de esta importante responsabilidad al dictar que obligatoriamente debe "contar con experiencia en la materia objeto de la Amexcid" y "haber desempeñado cargos afines a la CID" ${ }^{14} \mathrm{El}$ hecho de que la persona designada no acreditó tales atributos fue interpretado por varios funcionarios de la agencia como un retroceso en el proceso de atención a los estatutos de la ley.

Ahora bien, desde una perspectiva más alentadora, en 2013 la Amexcid diseñó un nuevo esquema institucional para intentar adecuarse a las ambiciosas metas planteadas. Si bien, en efecto, esta reestructuración administrativa se llevó a cabo, fue pragmática e inconclusa, dado a que no prosperó durante el sexenio la reforma al reglamento interno de la SRE necesaria para formalizarla. De esta particular situación resultó una agencia con dos organigramas: el legal u oficial (que aparece en la página en línea de la Amexcid, basado en el reglamento vigente de la cancillería) y el operativo o "real" (véase la figura 1), que fue el que funcionó en la práctica durante

${ }^{13}$ Margaret Karns y Karen Mingts, International Organizations. The Politics and Processes of Global Governance, Londres, Lynne Rienner Publishers, 2010, p. 38.

${ }^{14}$ Ley de Cooperación Internacional para el Desarrollo, Diario Oficial de la Federación, 6 de abril de 2011, art. 20, numerales 2 y 3. 
ese periodo. Si se considera la existencia de la Coordinación de Asesores, equiparable a una dirección general, la Amexcid se configuró como la instancia, con nivel de subsecretaría, más amplia de la SRE.

FIGURA 1

Organigramas oficial y operativo de la Amexcid

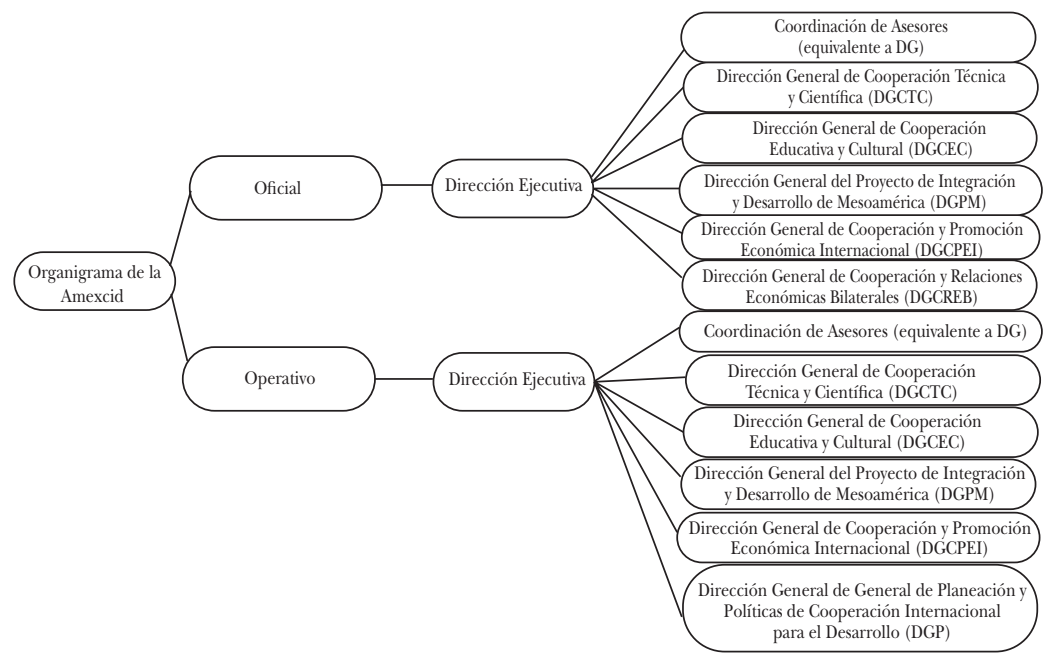

Fuente: elaboración propia con base en www.amexcid.gob.mx

Ahondando en el tema, es importante señalar que la Amexcid atendió de forma simultánea dos responsabilidades generales: por un lado, figurar desde luego como la instancia gestora y coordinadora, por excelencia, de la CID nacional. Por el otro, debido a que esta dependencia contó oficialmente con dos direcciones generales dedicadas a las relaciones económicas, ello hizo que la agencia constituyera también la faz de la diplomacia económica de la sRe. Por tanto, dado su considerable tamaño, junto con un confuso organigrama oficial/operativo, así como sus numerosos ámbitos de acción en rubros que de hecho rebasan la CID (como el fomento comer- 
cial, turístico e, incluso, gastronómico, así como los programas Proyecta 100000 y Proyecta 10 000), ${ }^{15}$ la Amexcid adquirió una desbordante dimensión y una estructura difusa, con la consecuente fragmentación. Ello le restó cohesión e identidad como instancia dedicada en exclusiva a la CID.

Asimismo, 93 de las cerca de 350 personas que laboran en la agencia (casi una cuarta parte) no tienen la condición de funcionarios de base del gobierno federal, sino que han sido contratadas de forma temporal bajo la figura de consultores externos del Programa de las Naciones Unidas para el Desarrollo (PNUD). La bolsa anual de este renglón, financiado por el gobierno de México, es de cerca de 1.8 millones de dólares. ${ }^{16}$ Tal circunstancia, que no es exclusiva del caso mexicano, sino que se reproduce en otros países, ${ }^{17}$ responde a la imposibilidad de abrir nuevas plazas en la estructura de la SRE-Amexcid por la negativa de la SHCP.

En materia financiera, el presupuesto ejercido por la Amexcid se desplazó de los 489.5 millones de pesos, en 2013, a 434 millones, en 2017 (el dato más reciente disponible); una disminución del 11.4\%, aunque si se considera sólo el lapso 2016-2017, la caída fue del $31.5 \%{ }^{18}$ Sobre este asunto, vale la pena destacar que, conforme escribió en 2017 el director ejecutivo de la agencia, "una vez descontados los gastos de operación, las cuotas a organismos internacionales y gastos por

${ }^{15}$ La DGCED alberga una Dirección General de Promoción Turística y Gastronómica. Los programas mencionados consisten en becas del gobierno para que mexicanos estudien inglés en Estados Unidos y Canadá, por lo que su ámbito escapa también al ejercicio de la CID.

16 A esto, hay que restar el cobro a la Amexcid por uso de cuenta, gastos administrativos y otros (overhead), del que el PNUD se beneficia, con un monto que oscila del $15 \%$ al $17 \%$ por cada transacción.

17 Por ejemplo, en Brasil, la proporción es del 45\%; en Uruguay, del $25 \%$, y en Colombia, del $16 \%$. Lo relevante es que existen experiencias en las que se logró reducir el número de personal con este estatus, a fin de lograr instituciones nacionales más consolidadas y autónomas. Tal es el caso de Argentina, cuya proporción pasó del 80\%, en 1993, al 5\%, en 2018.

18 Juan Pablo Prado Lallande, entrevista al embajador Agustín García López, director ejecutivo de la Amexcid, 26 de junio de 2018. 
traslado, el margen de acción presupuestaria [de la Amexcid] es en promedio $10 \%$ del presupuesto asignado originalmente". ${ }^{19}$ Yendo más allá de estos datos, y para contextualizar de mejor manera dicho presupuesto, si se considera que en 2017 la sRE ejerció un total de 484.7 millones de dólares, se desprende que el porcentaje de estos recursos que le correspondió a la agencia para gastos de operación fue de tan sólo el 1.7\%. Esta cifra, por sí misma, habla de que la CID no fue prioritaria para la cancillería, al menos en términos presupuestarios.

\section{Gráfica 1}

Presupuesto anual de la Amexcid, en millones de pesos (2013-2018)

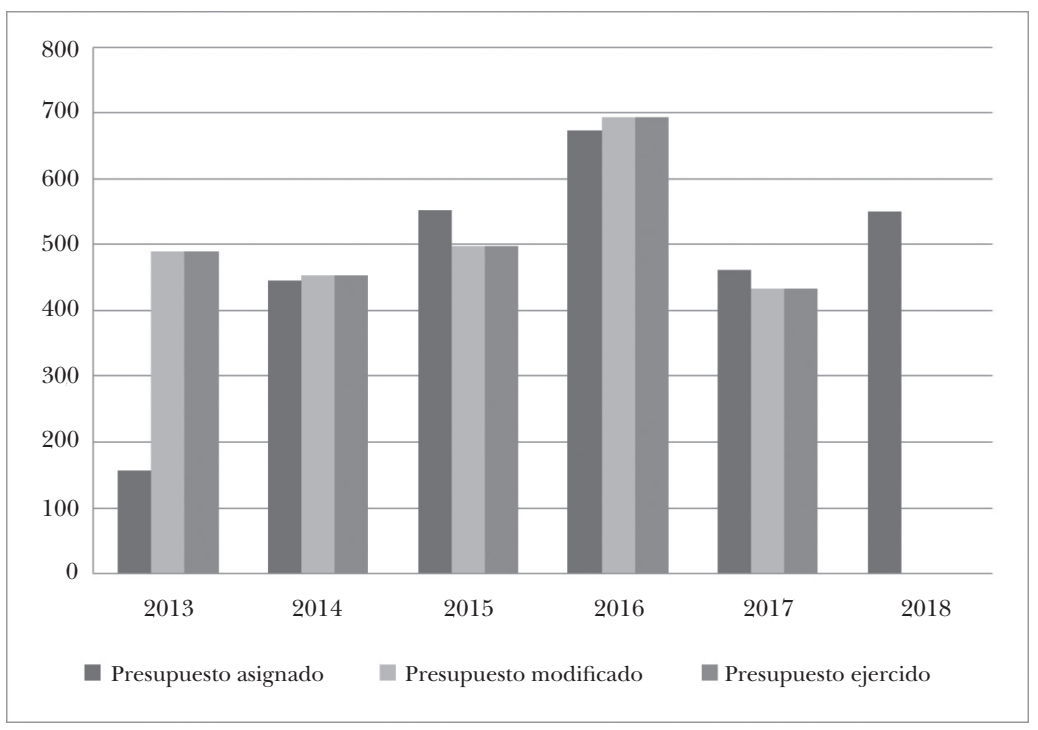

Fuente: Informe semestral del Consejo Consultivo Amexcid 2018.

Nota: para 2018 sólo se cuenta con el primer rubro graficado, que corresponde a presupuesto asignado.

${ }^{19}$ Agustín García-López Loaeza, "La cooperación mexicana para el desarrollo: el poder suave de la política exterior", Revista Mexicana de Política Exterior, 2017, núm. 111, p. 32. 
También en materia financiera, la Amexcid administró y coordinó diez fondos de cooperación internacional para el desarrollo, con los que se sufragó el costo de 111 proyectos, becas a extranjeros y acciones de ayuda humanitaria. De esos fondos, el más relevante es el Foncid, puesto que desde 2013 recibe recursos anuales por alrededor de cincuenta millones de pesos, de los cuales cerca de cuarenta millones tienen como destino el pago de las becas que el gobierno, vía Amexcid, concede a extranjeros para que estudien en México. El asunto es que estas fuentes monetarias no son predictibles: en 2017, la Cámara de Diputados omitió asignar presupuesto al Foncid, por lo que la agencia se vio en la necesidad de buscar financiamiento en las arcas de la cancillería para cubrir este vacío.

Pero si de fondos de cid se trata, por curioso que parezca, el más relevante de éstos en términos presupuestales y alcances no lo opera la Amexcid, sino la SHCP, dependencia que financia el Fondo de Infraestructura para Países de Mesoamérica y el Caribe o Fondo de Yucatán (FY). El FY se creó en 2011 para sustituir al Acuerdo de San José, cuyo órgano fiduciario fue competencia de Bancomext y cuyas decisiones relativas a su operatividad emanaron de un Comité Técnico formado por tres miembros de la SHCP y dos de la cancillería (uno de la Amexcid). Al amparo del Fy, en el sexenio se erogó la nada despreciable cifra de 134.7 millones de dólares, de los cuales el $79.8 \%$ se destinó a proyectos de infraestructura en Centroamérica y el 20.2\% aterrizó en el Caribe, para apoyar 17 proyectos en 12 países de ambas regiones. ${ }^{20}$

Si bien el Fy configura el instrumento más importante de la CID mexicana y llama la atención que, debido a que sus recursos provienen del pago de intereses por la venta de petróleo al amparo del extinto Acuerdo de San José, su fuente de financiación disminuye año tras año, con un remanente en 2018 de alrededor de 40 millones de dólares. De ahí que el director general del Proyecto de Integración y Desarrollo

${ }^{20}$ SRE, Sexto Informe de Labores 2017-2018, México, SRE, 2018, p. 169. 
de Mesoamérica de la Amexcid señaló que su financiamiento "no es sostenible". ${ }^{21}$

\section{LOS RESUlTADOS}

El Proyecto de Integración y Desarrollo de Mesoamérica o Proyecto Mesoamericano (PM), catalogado por la Amexcid como su estrategia insignia, ligado al $\mathrm{FY}$, es una relevante iniciativa de colaboración regional. Su fin es facilitar procesos de integración y desarrollo en los diez países participantes. ${ }^{22}$ Hasta febrero de 2017, los proyectos concluidos y en ejecución en el marco del PM tenían un valor de 35.4 millones de dólares. ${ }^{23}$

Otro aspecto de importancia son los informes sobre la CID que editan la Amexcid y la SRE, cuyos contenidos si bien son extensos y muestran mejoras en distintos rubros, los datos que los alimentan (en especial, la numeralia de proyectos de cooperación discriminados por tipología, destino, fuente, etc., y el número de becas asignadas) no siempre son consistentes ni dentro de un mismo informe, ni con respecto a ediciones previas o subsiguientes. ${ }^{24}$ Ello complicó, al director ejecutivo de la agencia, a las áreas políticas de la cancillería y al propio titular de la SRE, la toma de decisiones estratégicas relativas al uso dinámico de la cID como ejercicio programático y pragmático de política exterior.

${ }^{21}$ Juan Pablo Prado Lallande, entrevista al ministro Julián Juárez Cárdenas, director general del PM de la Amexcid, 26 de octubre de 2018.

${ }^{22}$ Belice, Colombia, Costa Rica, El Salvador, Guatemala, Honduras, México, Nicaragua, Panamá y República Dominicana.

23 Amexcid, "Proyecto de integración y desarrollo de Mesoamérica, años 2008-2017”, documento, 2017.

${ }^{24}$ Salta a la vista que, en el marco de una investigación, se encontró que ningún informe de la SRE ni de la Amexcid reportó el número de proyectos en el año 2015. Ante ello, la Amexcid señaló que ello se debe a los constantes cambios en la metodología aplicada y a la adecuación de sus bases de datos. 
En este mismo sentido, además del Procid, se dio otro significativo paso en el perfeccionamiento de la institucionalización de la CID mexicana: el Rencid. Por vez primera, y con base en lo dispuesto en la LCID, la Amexcid hizo público este registro nacional (del 2012 al 2017), cuyo contenido proviene de cálculos monetarios de la oferta de colaboración mexicana federal. El Rencid, como lo hacen los miembros del Comité de Asistencia al Desarrollo de la OCDE, incluye como recursos de CID las contribuciones que el gobierno federal aporta a organismos multilaterales, criterio que es cuestionable si se considera que buena parte de tales pagos son de orden contractual, esto es, de carácter obligatorio, como un compromiso legal e irrenunciable por el hecho de pertenecer a este tipo de organizaciones. De 2013 a 2017, la proporción promedio de estos aportes fue de $63 \%$ respecto al monto de financiación de toda la CID mexicana reportada en el Rencid en esos años, aunque en periodos clave, como en 2016, superó el $86 \%$.

Los datos del Rencid etiquetados como "Cooperación financiera” son también interesantes. Entre lo más significativo, destaca la condonación de deuda, vía la SHCP, a Cuba en 2013 por poco más de 340 millones de dólares, como expresión de un ejercicio innovador de política exterior para reconducir la relación México-La Habana, lastimada durante los dos sexenios anteriores. Sin embargo, los datos anuales ofrecidos por el Rencid sobre esta partida no parecen reflejar con exactitud los montos erogados por el gobierno del sexenio estudiado. Ejemplo de ello es que, por un lado, el registro señala que en materia de cooperación financiera, de 2013 a 2017, México aportó alrededor de 70 millones de dólares (exceptuando la citada condonación), mientras que en otro informe, ${ }^{25}$ la SRE indicó que mediante el FY se erogaron 134.7 millones de dólares en el mismo lapso, es decir, casi el doble. La Amexcid no ofreció respuesta al autor sobre tal incongruencia contable. Más allá de este asunto, en el sexenio de

$$
{ }^{25} \text { SRE, op. cit., p. } 169 .
$$


Peña Nieto, de acuerdo con el Rencid, la parte de la cooperación financiera fue de $25 \%$ del total de recursos.

En el plano educativo, también de conformidad con el Rencid, de 2013 a 2017, el acumulado de recursos de la cooperación educativa mexicana correspondió al $6 \%$ del total. En 2017, la cifra llegó a 21.5 millones de dólares, que se utilizaron para becar a 3883 estudiantes extranjeros en Méxi$\mathrm{Co}^{26} \mathrm{y}$, una cuantía menor a ésta para sufragar los gastos del innovador programa Escuelas México. ${ }^{27}$

La cooperación técnica y científica (CTC) que presta México es sustanciosa en cuanto a su número de proyectos, ${ }^{28}$ tanto en materia de oferta como de recepción, por la vía bilateral, regional o trilateral. Según cálculos elaborados en el marco de esta investigación, de 2013 a 2017, México instrumentó con sus socios un promedio anual de 160 proyectos. En 2017, la medición financiera de este esfuerzo arrojó un resultado aproximado de 14.5 millones de dólares. Esto posicionó al

${ }^{26}$ El Rencid incluye en esta partida recursos que la Secretaría de la Defensa Nacional y la Secretaría de Marina-Armada de México aportan en términos de becas ofrecidas a extranjeros para cursos militares en México, un ámbito que por definición no es propio de la CID. En consecuencia, tanto por esta razón como por otras inconsistencias, este dato de 2017 debe revisarse, pues si bien proviene de la Amexcid (a solicitud del autor), en un informe del Consejo Consultivo se señala que en ese año se ofrecieron 342 becas (más de 11 veces de un año a otro), sin mayor explicación de ello (Amexcid, Informe semestral del Consejo Consultivo Amexcid 2018, México, Amexcid, 2018, p. 33).

${ }^{27}$ Creado en 1996, Escuelas México trabaja en 17 países latinoamericanos y caribeños, con el objetivo de llevar a cabo mejoras en infraestructura y compra de material didáctico, que, con ello, fortalecen la imagen de México entre los beneficiarios de los casi 150 planteles educativos seleccionados. Desde ese entonces, este interesante programa ha auspiciado viajes a la capital mexicana para un total de 435 alumnos ganadores de concursos académicos y artísticos, así como para 320 profesores.

${ }^{28}$ Los datos cuantitativos del número de proyectos de cTC deben tomarse con cautela, dado a que, si bien constituyen un parámetro de análisis, no consideran aspectos cualitativos sobre la dimensión de tales ejercicios, costo e impacto, lo cual hace que sean disímiles y, por ende, no comparables entre sí. 
país como una potencia regional en el rubro de la CTC, lo cual se constata en el Informe de la Cooperación Sur-Sur en Iberoamérica, el cual manifiesta que México ocupó el segundo lugar como proveedor de proyectos de CTC/CSS en Latinoamérica (después de Argentina y antes que Brasil). ${ }^{29}$

\section{GRÁFICA 2}

Evolución del número de proyectos de cooperación técnica y científica de México, 2013-2018

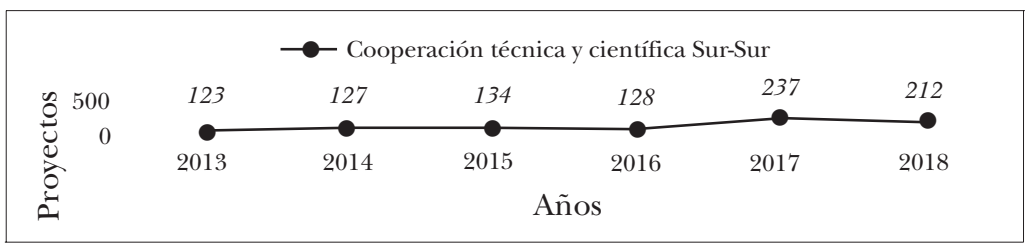

Fuente: elaboración propia, con base en los informes de labores de la SRE de los años 2013, 2014, 2016, 2017 y 2018.

Otro dato revelador es que de 2013 a 2018, el $70 \%$ de los proyectos de CTC mexicana se instrumentaron por la vía bilateral, mientras que el $16 \%$ se hizo mediante canales regionales y el $14 \%$ a través de mecanismos triangulares. Este indicador confirma que, conforme a la tendencia latinoamericana, la css de México de nueva cuenta se desplegó de manera preferencial mediante el esquema bilateral, como un claro y evidente mecanismo directo de política exterior. Conforme al Rencid, de 2013 a 2017, el esfuerzo humano y logístico de la CTC contabilizado en términos monetarios representó el 3\% del total de la financiación de la CID mexicana. Esto llama la atención, en virtud de que en 2011 y 2012 fue del $7 \%$. En cuanto a la ayuda humanitaria, la partida fue reducida, al no superar el $1 \%$.

29 SEGIB, Informe de la Cooperación Sur-Sur en Iberoamérica, Madrid, SEGIB, 2017, p. 17. 


\section{GráficA 3}

Cooperación Sur-Sur, técnica y científica de México por vía de suministro (2013-2018)

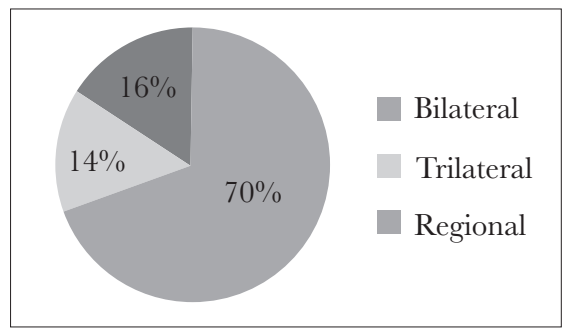

Fuente: elaboración propia, con base en los informes de labores de la SRE de los años 2013, 2014 y 2018, y los informes del Consejo Consultivo de la Amexcid de los años 2015, 2017 y 2018.

\section{GrÁFICA 4}

Porcentajes de la financiación de la cooperación internacional para el desarrollo de México por rubros (2013-2018)

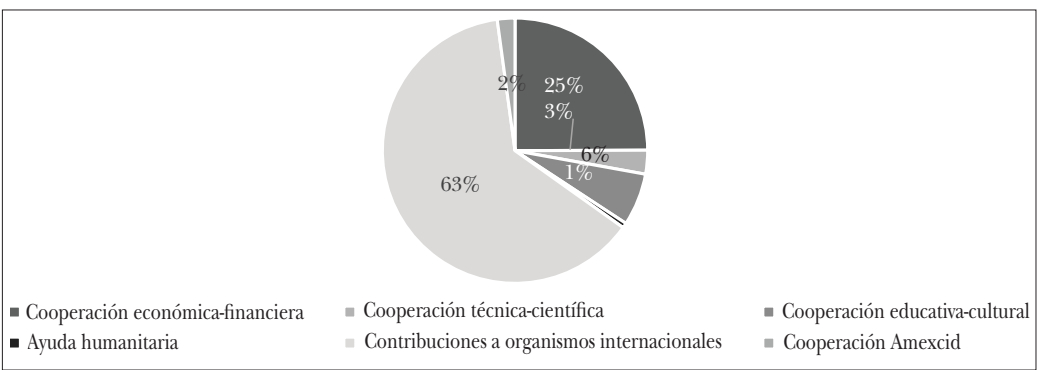

Fuente: elaboración propia, con base en los informes de labores de la SRE de los años 2013, 2014 y 2018, y los informes del Consejo Consultivo de la Amexcid de los años 2015, 2017 y 2018. 


\section{GRÁFICA 5}

Evolución anual de la financiación de la cooperación internacional para el desarrollo de México por rubros (2013-2018)

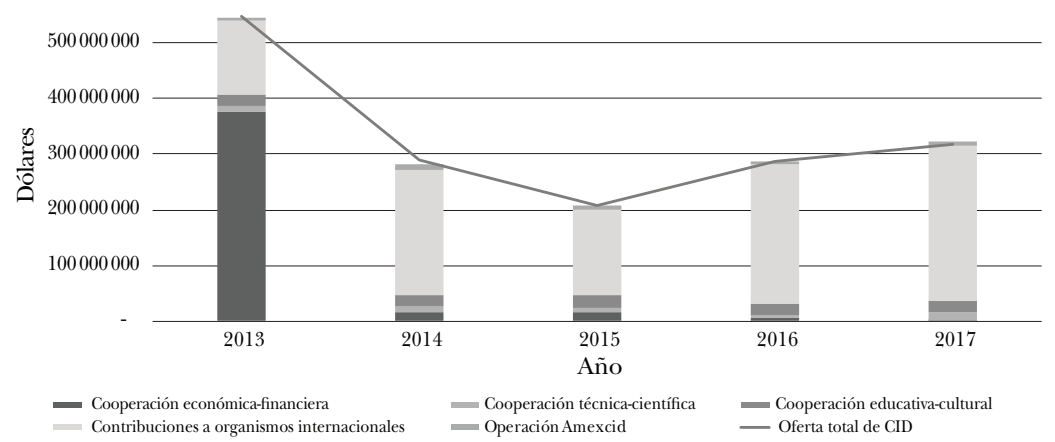

Fuente: elaboración propia con base en el documento de la Amexcid, "Cuantificación de la cid de México", México, Secretaría de Relaciones Exteriores, SRE, https://infoamexcid.sre.gob.mx/amexcid/ccid 2013/ho me.html (consulta del 28 de abril de 2017).

Al sumar las citadas modalidades comprendidas en el Rencid, es interesante señalar que mientras que en 2013 el esfuerzo monetario nacional respectivo alcanzó los 548.3 millones de dólares, en 2017 el monto fue de 317.6 millones de dólares, arrojando un descenso del 43\%, que, como se verá más adelante, se explica por el debilitamiento de los factores estructurales que condicionan el dinamismo de la CID (véase la gráfica 5). Ahora bien, al excluir la citada condonación de deuda a Cuba, el pago a organismos multilaterales y los gastos administrativos de la Amexcid (esto es, considerando exclusivamente las modalidades más "finas" de la oferta federal de CID, mediante el esquema de css), el gasto pasó de 66 millones de dólares, en 2013, a 37.5 millones de dólares, en 2017, de lo que resulta una disminución de $44 \%$.

Utilizando este mismo criterio contable (esto es, al sumar la cooperación financiera, la educativa y cultural, la técnica y científica y la ayuda humanitaria), cálculos propios 
con base en el Rencid muestran que la CID mexicana de 2013 a 2017, equiparable a su css, sumó 233 millones de dólares, esto es, un promedio de 46 millones de dólares por año. El balance general financiero de la cooperación internacional para el desarrollo, conforme a los datos totales del Rencid en sus distintos rubros, es que, por un lado, de 2013 a 2017, México utilizó 1650 millones de dólares para sufragar su oferta de GID, mientras que en calidad de receptor de asistencia oficial para el desarrollo (proveniente de donantes tradicionales y organismos multilaterales) se benefició con 2508 millones de dólares, lo cual le generó un "superávit" de 858 millones de dólares en ese periodo de tiempo. ${ }^{30} \mathrm{De}$ esto resulta que, en promedio, por cada dólar que la Amexcid tasó como financiamiento de oferta de CID por conducto de la contabilización de sus ejercicios de css, se benefició con 1.52 dólares provenientes del extranjero. Si se compara la recepción de asistencia oficial para el desarrollo (AOD) de 2013 a 2017 (2508 millones de dólares) frente a la oferta de css mexicana en términos monetarios (233 millones de dólares), la relación es de cerca de 10.7 a 1. Esto constata que el perfil de México en este segmento de la CID se configura de forma predominante como beneficiario y no como oferente de css.

Por último, en la administración de Peña Nieto resalta un avance cualitativo de la CID mexicana: el activismo político por parte de México en los organismos y foros multilaterales del área. La referida AGCED, el Foro de CID de Naciones Unidas y el Comité de css de la cEPAL son algunos ejemplos de espacios en donde México, mediante la Amexcid, impulsó un diálogo constructivo, en pro de una gobernanza de la CID más incluyente.

30 Amexcid, "Discurso del director ejecutivo de la Amexcid. XIII Sesión Ordinaria del Consejo Consultivo de la Amexcid", Ciudad de México, 23 de noviembre, 2018, p. 1. 


\section{Consideraciones finales}

En un efervescente ámbito internacional que se debate entre el conflicto, la competencia y la colaboración, el ejercicio de la CID constituye una herramienta de política exterior de obligada referencia, en particular para aquellos países que, como México, pretenden ejercer un rol proactivo en su entorno regional y global. En este sentido, las relaciones y, por ende, la colaboración Sur-Sur suponen un cambio incremental y de alcance transformador del sistema internacional, ${ }^{31}$ por lo que México y otros países en desarrollo intentan asumir responsabilidades adicionales en ese ámbito, en aras de sumarse a esta dinámica mundial.

En ese camino, durante el transcurso del gobierno de Enrique Peña Nieto, y como un subelemento de su política exterior, el país concretó una variedad de ejercicios de CID, de cuyo andamiaje institucional y características fácticas más representativas dio cuenta este artículo. Al inicio de su mandato, en un entorno favorable tanto a nivel nacional como internacional, el gobierno renovó las expectativas de ejercer una política exterior y, de forma inherente, una CID afines al precepto de hacer de México un país con responsabilidad global. El ambicioso mandato del PND en cuanto a que la política exterior se basaría en la cooperación internacional para el desarrollo es el ejemplo más contundente a ese respecto. Para ello, se pusieron en marcha acciones concretas a fin de mejorar la institucionalidad de la colaboración mexicana, entre las cuales sobresalen una reestructuración pragmática, aunque inconclusa, de la Amexcid; la publicación, por vez primera, del Procid; el Rencid, y la activación del Foncid; todos ellos, encomiables avances que robustecen al arduo proceso de construcción de una política pública de CID.

A partir de entonces, el gobierno mexicano mantuvo -e incluso incrementó- su potencial para desplegar acciones

31 Élodie, Brun, El cambio internacional mediante las relaciones Sur-Sur, México, El Colegio de México, 2018, p. 27. 
de CID mediante vías innovadoras, teniendo como referente geográfico prioritario Centroamérica, en clara conciliación con los objetivos de política exterior hacia el sur y con base en lo dispuesto por la LCID. En este sentido, además de una prolija gama de proyectos de CTC y becas, por su peso financiero y efectos generados, sobresalieron el FY y el PM, que se constituyeron como los mecanismos regionales insignia de la oferta de CID en el sexenio de Peña Nieto.

Empero, a partir de 2017, esos esfuerzos enfrentaron un contexto interno ${ }^{32}$ y externo que no favoreció el ejercicio de una política exterior activa e integral, debilitando, en consecuencia, los factores estructurales de los cuales depende la CID, abordados en la primera parte del artículo. En el plano internacional, destaca la elección de Donald Trump en la presidencia de Estados Unidos, lo que ocasionó que la política exterior mexicana se tornara reactiva, con el objeto de defender -como no podría ser de otra manera- los intereses mexicanos en ese país, y que los esfuerzos políticos y financieros de la cancillería se enfocaran en la frontera norte. ${ }^{33}$

Estos factores incidieron de forma negativa en el peso específico y político de la CID en la SRE, condicionando el proceso que se estaba desarrollando en favor de una institucionalidad más propicia para la Amexcid y su adecuado financiamiento, el cual, de 2013 a 2017, cayó en 43\%. Ello ralentizó la capacidad de la agencia para configurarse como un facilitador más significativo al servicio de la política exte-

32 Debido, en esencia, a la corrupción que lastimó a la figura presidencial, la inseguridad, la priorización de las reformas estructurales y la reducción del presupuesto federal.

33 Una experiencia en esa dirección fue el aporte extraordinario por mil millones de pesos que en 2016 hizo la shcP a los consulados de México en Estados Unidos. Ello coincide con el planteamiento de que la CID compite en desventaja con las demás prioridades de la política exterior. Véase Jorge Schiavon y Bruno Figueroa, "Las capacidades de la política exterior de México: diagnóstico (2000-18) y propuestas (2018-24)", en Jorge Schiavon et al. (eds.) La política exterior de México 2018-2024: diagnóstico y propuestas, México, CIDE-UnANL, 2018, p. 22. 
rior, tal y como el gobierno se había comprometido a hacerlo (quizás con un exacerbado énfasis) al inicio del sexenio y conforme lo estipula la ley.

Como consecuencia, se detectan dos implicaciones generales; una de corte crítico y la segunda de orden propositivo. Por un lado, en el periodo de Peña Nieto, más allá de los inobjetables esfuerzos y alentadores avances reseñados, la CID careció de medios políticos, institucionales y financieros adecuados para tener mayor impacto, conforme a lo inicialmente planeado. Ello hizo complejo que desde la dirección de la Amexcid la política exterior se basara en la CID.

Por otro lado, la agencia, incluso sin contar con las condiciones ideales y recurriendo al máximo de sus capacidades operativas, además de coordinar acciones de colaboración internacional, avanzó, en la medida de sus posibilidades, en perfeccionar la institucionalidad de la CID (activación del Procid, Simexcid, Rencid y Foncid), una complicada tarea que es menester reconocer en su adecuada dimensión.

Lo expuesto en el artículo, sin demeritar los avances sintetizados, explica diversas inconsistencias en la conducción de la CID durante estos últimos seis años, entre las cuales destacan las siguientes: la Amexcid, equiparable a la subsecretaría más grande de la SRE, sin cohesión y con instancias desvinculadas de la CID, no fue objeto de suficientes adecuaciones internas legales, adaptadas a los objetivos programáticos establecidos en 2013 en la legislación que la creó. En este sentido, también cabe mencionar que no sólo el presupuesto de la Amexcid disminuyó en $11.4 \%$ de 2013 a 2017, sino que los recursos destinados a la agencia fueron una ínfima parte del presupuesto de la cancillería (por ejemplo, del $1.7 \%$ en 2017). Una cuantía como ésta hace complejo que la política exterior, a través del liderazgo de la Amexcid, se base en la CID, conforme lo dictó el PND.

En esa misma dinámica, según el Rencid, los montos totales de la CID mexicana disminuyeron en 43\% de 2013 a 2017. $\mathrm{Al}$ excluir la condonación de deuda a Cuba llevada a cabo por la SHCP, los pagos a organismos internacionales y los gas- 
tos operativos de la Amexcid (es decir, considerando los rubros más precisos de la CID vía css), la bolsa mexicana anual promedio fue de 46 millones de dólares, que en conjunto suman aportaciones por 233 millones de dólares en el periodo considerado. De 2013 a 2017, la proporción entre la recepción de asistencia oficial para el desarrollo (2508 millones de dólares) y el otorgamiento de css en términos monetarios (233 millones de dólares) fue de casi 11 a 1. Estos datos evidencian que la posición de México en el ámbito de la CID corresponde, de forma predominante, a la recepción y no a la oferta, al menos en términos cuantitativos.

Asimismo, una tercera parte del personal de este organismo desconcentrado de la SRE labora bajo la figura de consultor del PNUD, ejercicio administrativo de triangulación que ocasiona que, conforme a fuentes consultadas en la Amexcid, alrededor de 1.8 millones de dólares del erario nacional en el sexenio se utilizaran para financiar servicios de manejo de cuenta a favor de ese organismo de las Naciones Unidas, un monto equiparable a lo que el Foncid recibió en dos años. Siguiendo el hilo monetario, irónicamente, las fuentes del FY -por mucho, el mecanismo de cooperación más activo del gobierno de México en este sexenio- son insostenibles, en virtud de que sus recursos se agotan y se carece de fuentes alternas para sufragarlo a futuro. Ahondando en las inconsistencias de la cooperación mexicana, Bruno Figueroa es enfático al señalar que "la sRe nunca tuvo el monopolio de la cooperación internacional", 34 lo cual obedece a la siguiente paradoja, que coincide con lo descrito en este artículo: la cooperación técnica gestionada por la SRE cuenta con escasos recursos y tiene poco impacto, mientras que la colaboración financiera, más robusta (como el FY y la condonación de deuda), es conducida por la SHCP y no por la cancillería.

${ }^{34}$ Bruno Figueroa Fischer, Cien años de cooperación internacional de México, 1900-2000: solidaridad, intereses y geopolítica, México, SRE-IMR-Acervo Histórico Diplomático, 2016, p. 393. 
Si bien México despliega una amplia gama de ejercicios de CID, varios de ellos mediante estrategias innovadoras y con efectos transformadores, la Amexcid no consiguió poner en marcha una adecuada estrategia de difusión de su labor, que comunicara su pertinencia e impacto, tanto en la propia cancillería como entre los otros poderes (en especial, el Legislativo), así como a la población en general ${ }^{35} \mathrm{y}$, desde luego, en el extranjero, en términos de lograr una visibilidad positiva de México (y, en consecuencia, de poder suave).

La enseñanza que deja el sexenio 2012-2018 es que la Amexcid ostenta una responsabilidad de mayor magnitud y complejidad de lo que los impulsores de la LCID y los tres secretarios de Relaciones Exteriores del periodo diseñaron e interpretaron, respectivamente. Adicionalmente a lo anterior, la ansiada reestructuración de la agencia no se consolidó, mientras que el declinante financiamiento recibido no le permitió, a la altura de lo planteado, cumplir con mayor oportunidad las expectativas generadas por las propias autoridades en el ramo. En una frase, las deficitarias capacidades políticas, institucionales y financieras asignadas en este sexenio a la Amexcid, que obedecieron a un contexto nacional e internacional adverso, afectaron su capacidad de cumplir las ambiciosas responsabilidades que le fueron conferidas al inicio del gobierno de Peña Nieto para hacer de la CID un instrumento más tangible de la política exterior.

Al fin y al cabo -y como ocurre en prácticamente todo Estado y, en especial, en periodos de recortes presupuestales y disminución o concentración del activismo internacional en otros frentes-, la cooperación internacional para el desarrollo no suele ser la principal herramienta de política exte-

${ }^{35} \mathrm{Si}$ bien la cid cuenta con una alta aprobación entre el público y los líderes de opinión en México (83\% y 99\%, respectivamente), el primero la identifica, en especial, como "un medio para promover la paz" y "otorgar ayuda humanitaria" (Gerardo Maldonado et al., Los mexicanos ante los retos del mundo: opinión pública, líderes y política exterior. México, las Américas y el mundo, 2016-2017, México, CIDE, 2018, p. 92), percepción que en términos conceptuales no es precisa. 
rior de los países, sino, por el contrario, es con frecuencia sacrificada a favor de otras prioridades. Esto es lo que explica (mas no justifica) que, como se sustenta en este estudio, la CID mexicana no lograse escalar suficientes peldaños como estrategia clave de las relaciones internacionales del gobierno durante el periodo estudiado.

En definitiva, en un convulso contexto que se desplazó de una situación propicia a una compleja y que afectó a la política exterior y, en consecuencia, a los factores estructurales que explican la dinámica de la CID, el análisis de la cooperación mexicana en el gobierno del presidente Enrique Peña Nieto arrojó alentadores avances, aunque también inconsistencias, teniendo como referencia los ambiciosos objetivos inicialmente planteados.

\section{Bibliografía}

Amexcid, "Cuantificación de la cid de México", México, Secretaría de Relaciones Exteriores, SRE, https://infoamexcid.sre.gob. $\mathrm{mx} / \mathrm{amexcid} / \mathrm{ccid} 2013 /$ home.html (consulta del 28 de abril de 2017).

Amexcid, "Discurso del director ejecutivo de la Amexcid. XIII Sesión Ordinaria del Consejo Consultivo de la Amexcid", documento, 23 de noviembre de 2018.

Amexcid, Informe Anual del Consejo Consultivo Amexcid 2015, México, Amexcid, 2015.

Amexcid, Informe Anual del Consejo Consultivo Amexcid, 2017, México, Amexcid, 2017.

Amexcid, Informe semestral del Consejo Consultivo Amexcid 2018, México, Amexcid, 2018.

Amexcid, "Proyecto de integración y desarrollo de Mesoamérica, 2008-2017", documento, Amexcid, 2017.

BRUn, Élodie, El cambio internacional mediante las relaciones Sur-Sur, México, El Colegio de México, 2018. 
Borbolla Compé́n, Daniela, "La construcción institucional de la Amexcid", Revista Mexicana de Política Exterior (2014), núm. 102, pp. 55-70.

Figueroa Fischer, Bruno, Cien años de cooperación internacional de México, 1900-2000: solidaridad, intereses y geopolítica, México, SRE-IMR- Acervo Histórico Diplomático, 2016.

García-López LoAeza, Agustín, "La cooperación mexicana para el desarrollo: el poder suave de la política exterior", Revista Mexicana de Política Exterior (2017), núm. 111, pp. 21-39.

Gobierno de la República, Plan Nacional de Desarrollo 2013-2018, México, Gobierno de la República, 2013.

Green, Rosario, "Política exterior de Estado con los tres órdenes de gobierno", El Universal, 1 de agosto de 2012.

Karns, Margaret y Karen Mingts, International Organizations. The Politics and Processes of Global Governance, Londres, Lynne Rienner Publishers, 2010.

Ley de Cooperación Internacional para el Desarrollo, Diario Oficial de la Federación, 6 de abril de 2011

Maldonado, Gerardo, Karen Marín, Guadalupe González y Jorge A. Schiavon, Los mexicanos ante los retos del mundo: opinión pública, líderes y política exterior. México, las Américas y el mundo, 2016-2017, México, CIDE, 2018.

Meade Kuribreña, José Antonio, "México y la cooperación internacional para el desarrollo", Revista Mexicana de Política Exterior (2014), núm. 102, pp. 5-6.

Milani, Carlos R.S, Solidariedade e Interesse: motivações e estratégias na cooperação internacional para o desenvolvimento, Curitibba, Brasil, Appris Editora, 2018.

Nye JR., Joseph S., El poder suave. La clave del éxito en la política internacional, México, Universidad Iberoamericana, 2016.

oEcD, odA, Receipts and Selected Indicators for Developing Countries and Territories, París, OECD-DAC, 2018.

Prado Lallande, Juan Pablo, entrevista al embajador Agustín García López, director ejecutivo de la Amexcid, 26 de junio de 2018.

Prado Lallande, Juan Pablo, entrevista al ministro Julián Juárez Cárdenas, director general del PM de la Amexcid, 26 de octubre de 2018. 
Prado Lallande, Juan Pablo, "La cooperación internacional para el desarrollo de México", Revista Mexicana de Ciencias Políticas y Sociales (2014), núm. 222, pp. 51-86.

Prado Lallande, Juan Pablo, "Mexico's Role in Development Cooperation: Bridging North and South", en Rahul Chandran y Hannah Cooper (eds.), Meeting the Challenges of the 2030 Agenda: Alternative Forms of Development Cooperation and the United Nations, Tokyo, United Nations University, 2015.

Schiavon, Jorge, y Bruno Figueroa, "Las capacidades de la política exterior de México: diagnóstico (2000-2018) y propuestas (20018-24)", en Schiavon, Jorge, Rafael Velázquez Flores y Humberto Garza Elizondo (eds.), La política exterior de México 2018-2024: diagnóstico y propuestas, México, CIDE-UnANL, 2018.

Secretaría de Relaciones Exteriores, SRE, Cuarto informe de labores 2015-2016, México, SRE, 2016.

Secretaría de Relaciones Exteriores, SRE, Primer informe de labores 2012-2013, México, SRE, 2013.

Secretaría de Relaciones Exteriores, SRE, Quinto informe de labores 2016-2017, México, SRE, 2017.

Secretaría de Relaciones Exteriores, SRE, Segundo informe de labores 2014-2014, México, SRE, 2014.

Secretaría de Relaciones Exteriores, SRE, Sexto Informe de labores 2017-2018, México, SRE, 2018.

Secretaría de Relaciones Exteriores, SRE, Tercer informe de labores, 2014-2015, México, SRE, 2015.

SEGIB, Informe de la cooperación Sur-Sur en Iberoamérica, Madrid, SEGIB, 2017. 
